\title{
Antiviral treatment for COVID-19: the evidence supporting remdesivir
}

\author{
Authors: Charlotte Richardson, ${ }^{A}$ Sanjay Bhagani, ${ }^{B}$ and Gabriele Pollara ${ }^{C}$
}

The emergence of the novel beta coronavirus SARS-CoV-2 and the ensuing COVID-19 pandemic has generated a rapidly evolving research landscape in the search for new therapeutic agents. The intravenous antiviral drug remdesivir has in vitro activity against SARS-CoV-2 and now studies have reported its clinical efficacy, demonstrating shorter time to recovery in hospitalised patients with severe COVID-19. Adverse event rates were low and remdesivir has now received conditional marketing authorisation from the European Medicines Agency. An interim clinical commissioning policy is in place in the UK. These studies make remdesivir the first antiviral drug able to alter the natural history of severe COVID-19, and a benchmark for the comparison of new therapies in the future. Ongoing studies are investigating its use in early $\mathrm{mild} / \mathrm{moderate}$ COVID-19, alternative formulations, and the combination of remdesivir with immunomodulatory agents.

KEYWORDS: COVID-19, remdesivir, antiviral

DOI: $10.7861 /$ clinmed.2020-0524

\section{Introduction}

Since the emergence of the novel beta coronavirus SARS-CoV-2 (severe acute respiratory syndrome coronavirus 2) and the ensuing COVID-19 pandemic, several clinical trials have been initiated globally to rapidly evaluate possible therapeutics. Remdesivir is an RNA polymerase inhibitor with broad antiviral activity against several RNA virus families.' Its safety profile in humans has been established through trials in healthy volunteers and patients with Ebola virus. ${ }^{2}$ Other therapeutics proved more effective in Ebola and thus remdesivir never received approval for clinical use prior to the emergence of COVID-19. Remdesivir inhibits in vitro replication of SARS-CoV-1 and MERS-CoV (other beta coronaviruses that cause respiratory disease in humans), ${ }^{3}$ and has clinical efficacy

Authors: Aspecialist registrar in infectious diseases, Royal Free London NHS Foundation Trust; ${ }^{B}$ consultant physician in infectious diseases, Royal Free London NHS Foundation Trust, and honorary senior

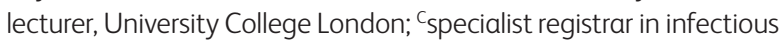
diseases, Royal Free London NHS Foundation Trust, and Wellcome Trust postdoctoral clinical research fellow and NIHR clinical lecturer, University College London

\section{Key messages}

> The preliminary results of the Adaptive Covid-19 Treatment Trial (ACTT-1) international randomised controlled trial demonstrated a significant reduction in median time to recovery of 4 days with remdesivir compared to placebo in hospitalised patients requiring supplemental oxygen. No significant effect on mortality was observed.

$>$ An international randomised trial of treatment duration demonstrated no difference in clinical outcomes between 5- and 10-day courses of remdesivir.

> Remdesivir is administered intravenously and has been shown to be well tolerated, with the most common adverse events being anaemia, deranged liver function tests, impaired renal function and hyperglycaemia.

> Remdesivir received a conditional marketing authorisation for use in the EU from the European Medicines Agency on 3 July 2020, applicable in the UK during the post-Brexit transition period. An interim clinical commissioning policy is in place.

> Current limitations include IV formulation and contraindication in renal impairment and patients with liver enzyme elevation

> Ongoing studies are investigating the combination of remdesivir and various immunomodulatory agents, use of alternative formulations and use in early mild/moderate COVID-19.

in animal models of these infections. ${ }^{3,4}$ Moreover, remdesivir has antiviral activity in vitro against SARS-CoV-2, and this finding prompted interest in its potential clinical efficacy in COVID-19. 5

\section{Clinical trials}

The first published double-blind placebo-controlled randomised controlled trial (RCT) investigating the use of remdesivir in COVID-19 was a multicentre study in China. ${ }^{6}$ Only 237 of the planned 453 patients were recruited due to declining case numbers. The study aimed to identify a difference in the primary endpoint of time to clinical improvement up to day 28 after randomisation. Hospitalised patients with laboratory-confirmed SARS-CoV- 2 with both oxygen saturations $\leq 94 \%$ on air and radiological changes were randomised to remdesivir ( $200 \mathrm{mg}$ on day 1 followed by $100 \mathrm{mg}$ on days 2-10) or placebo. Remdesivir was not associated with a difference in achieving the primary 
endpoint (hazard ratio 1.23, 95\% confidence interval [CI] 0.871.75), and there was also no difference in the secondary endpoints of mortality or time to viral undetectability in nasopharyngeal swabs and sputum specimens. Interestingly, remdesivir was associated with a 5 -day reduction in time to clinical improvement if administered within 10 days of symptom onset, in contrast to a 1-day reduction if administered after 10 days. Although not significant, this finding suggested benefit to initiating treatment early in the disease course, when viral loads are highest.?

More recently, the Adaptive Covid-19 Treatment Trial (ACTT-1) international RCT reported its preliminary findings. ${ }^{8}$ This study compared 10 days remdesivir to placebo in a cohort of hospitalised SARS-COV-2-positive adult patients with a wide disease spectrum at baseline. Randomisation resulted in 538 and 521 patients recruited in the remdesivir and placebo arms respectively, which were then included in the intention-to-treat analysis. The primary endpoint was originally defined as difference in clinical status at day 15 , but subsequently changed during the study to time to recovery up to day 28 , on the basis of emerging data suggesting a more protracted COVID-19 disease course than originally envisioned.

Findings were analysed at the time of planned interim review when $80 \%$ of the recruited patients had completed the 28 -day enrolment period. The two arms were well matched, with average age $58,64 \%$ male and comparable ethnicity and comorbidity. Median time from symptom onset to randomisation was 9 days (interquartile range 6-12), reflecting the COVID-19 disease course and timing of progressive disease leading to hospitalisation seen in other studies. ${ }^{9}$ Clinical status at baseline was scored on an 8-point ordinal scale and the majority of patients were in the group requiring supplemental oxygen, without need for high-flow oxygen or ventilation.

The ACTT-1 study identified a reduction in median time to recovery of 4 days with remdesivir compared to placebo (11 vs 15 days respectively), with a rate ratio for recovery of 1.32 (95\% CI 1.12-1.55, $p<0.001$ ) whereby a value $>1$ indicates a benefit for remdesivir. This reduction was most significant in hospitalised patients requiring supplemental oxygen, without need for highflow oxygen or ventilation. 14-day mortality was lower in the remdesivir group, but the difference was not statistically significant (hazard ratio $0.70,95 \%$ CI $0.47-1.04$ ), and 28-day mortality data were not available at the time of publication. No difference in the effect of remdesivir was noted when comparing initiation before or after 10 days from symptom onset. Although a longer follow up publication is planned, early unblinding and the opportunity to transfer patients from placebo to treatment will reduce power to detect a mortality benefit.

Another international RCT randomised 397 patients to compare 5 vs 10 days of remdesivir in patients with severe COVID-19.10 This study showed no difference in improvement in clinical status at day 14 , suggesting 5 days of therapy to be sufficient for most patients with severe disease. As the trial did not include a placebo arm, the absolute clinical benefit of remdesivir could not be determined in this study. More recently, a comparative analysis demonstrated greater clinical recovery and lower mortality in hospitalised patients receiving remdesivir (Gilead SIMPLE 5773 study) compared to a matched cohort receiving standard of care (Gilead 5807 study). ${ }^{11}$

However, this was not a randomised study, instead making use of a comparable control population, and thus unadjusted confounders could not be excluded. Finally, the efficacy of remdesivir has also been investigated for moderate COVID-19 disease in an open-label RCT (Gilead SIMPLE 5774 study), which demonstrated improved clinical status at day 11 in those randomised to 5 days of remdesivir compared to standard care. While significant, the effect size was small and was not seen in those randomised to 10 days remdesivir, with no difference in mortality shown. ${ }^{12}$

Safety data across all studies have shown remdesivir to be well tolerated, with the most common adverse events being anaemia, deranged liver function tests, impaired renal function and hyperglycaemia. The most frequently reported severe adverse effect was respiratory failure, also a recognised complication of severe COVID, further supporting the safety profile of remdesivir.

\section{Clinical context}

Remdesivir was the first antiviral therapy to demonstrate significant clinical benefit for COVID-19 in the context of a RCT. On 26 May 2020 the UK Medicines and Healthcare products Regulatory Agency (MHRA) gave a positive scientific opinion for use of remdesivir in severe COVID-19 disease requiring supplemental oxygen, leading to its availability via the Early Access to Medicine scheme (EAMS). Remdesivir (marketed as Veklury) received a conditional marketing authorisation for use in the EU from the European Medicines Agency on 3 July 2020. This authorisation also applies in the UK during the post-Brexit transition period to December 2020, with an interim clinical commissioning policy in place to define routine access to remdesivir which replaces the EAMS (Box 1). Secondary criteria are included to be used should there be limitations in the supply of remdesivir in the UK. ${ }^{13}$

The reported clinical benefits described above, predominantly a reduction in recovery time, may have significant impact on reducing hospital bed requirements, particularly during peaks of COVID-19 activity. Nevertheless, it needs to be acknowledged that the clinical benefits are modest, particularly in the absence of data supporting reduced mortality. Moreover, the clinical indication for which efficacy has been demonstrated remains narrow, namely hospitalised patients, excluding the majority of individuals infected with COVID-19 who never require admission. In addition, it is not yet clear whether early administration of remdesivir in $\mathrm{mild} /$ moderate disease may prevent hospital admission, clinical deterioration or impact on transmission.

\section{Box 1. NHS interim clinical commissioning policy:} Remdesivir for patients hospitalised with COVID-19 (adults and children aged 12 years and older) ${ }^{13}$

Eligibility criteria (in accordance with the product licence):

> Hospitalised with COVID-19

> With pneumonia requiring supplemental oxygen

> Adults, and adolescents $\geq 12$ years of age and $\geq 40 \mathrm{~kg}$

$>\mathrm{eGFR} \geq 30 \mathrm{ml} / \mathrm{min}$

> Alanine aminotransferase (ALT) below 5 times the upper limit of normal at baseline

Remdesivir should be avoided in pregnancy unless clinicians believe the benefits of treatment outweigh the risks to the individual. 
These limitations in the impact of remdesivir are pertinent in the face of two recent developments. First, while remdesivir had been provided for free through EAMS, post-licensing costs are estimated to be $£ 2,000$ per patient, ${ }^{14}$ which will force many healthcare providers to make challenging cost-benefit calculations in the near future relating to implementing remdesivir in local treatment guidelines. Second, other therapeutic strategies have now shown clinical benefit in COVID-19. Most strikingly, a 10-day course of oral dexamethasone reduced mortality in severe COVID-19. ${ }^{15}$ Combining the antiviral properties of remdesivir with the immunomodulatory activity of steroids seems plausible, but this combination has not been formally investigated in terms of clinical benefit.

\section{Future research and conclusions}

The use of remdesivir in COVID-19 continues to be the focus of ongoing investigation, and it remains one of the treatment arms being compared to standard of care in the WHO Solidarity trial. The clinical benefit observed to date has also obligated its inclusion into standard care for newer studies on ethical grounds. These are focusing on combination of antiviral properties of remdesivir with immunomodulatory agents, the Janus kinase inhibitor baricitinib (ACTT-2 trial - NCT04401579) and the IL-6 receptor blocking monoclonal antibody tocilizumab (REMDACTA NCT04409262)

The use of remdesivir will remain limited by many factors, including its intravenous preparation and contraindication in renal impairment and in those with liver enzyme elevation above five times the upper limit of normal. Alternative formulations are required to overcome the barriers associated with IV medications, and also to circumvent the vehicle content preventing its use in renal impairment. Nebulised forms of remdesivir have entered clinical testing (communication from Gilead Inc) and may find use in these populations, as well as in patients with moderate COVID-19 with the aim of preventing hospital admission. Nevertheless, for now, intravenous remdesivir has become an important tool to improve outcomes for COVID-19 disease in hospitalised patients with pneumonitis requiring supplemental oxygen therapy.

\section{References}

1 Lo MK, Jordan R, Arvey A et al. GS-5734 and its parent nucleoside analog inhibit Filo-, Pneumo, and Paramyxoviruses. Sci Rep 2017; 7:43395.

2 Mulangu S, Dodd LE, Davey RT et al. A randomized, controlled trial of ebola virus disease therapeutics. N Engl J Med 2019;381: 2293-303.
3 Sheahan TP, Sims AC, Graham RL et al. Broad-spectrum antiviral GS-5734 inhibits both epidemic and zoonotic coronaviruses. Sci Transl Med 2017;9:eaal3653.

4 De Wit E, Feldmann F, Cronin ] et al. Prophylactic and therapeutic remdesivir (GS-5734) treatment in the rhesus macaque model of MERS-CoV infection. Proc Natl Acad Sci USA 2020;117:6771-6.

5 Wang M, Cao R, Zhang L et al. Remdesivir and chloroquine effectively inhibit the recently emerged novel coronavirus (2019-nCoV) in vitro. Cell Res 2020;30:269-71.

6 Wang $Y$, Zhang D, Du G et al. Remdesivir in adults with severe COVID-19: a randomised, double-blind, placebo-controlled, multicentre trial. Lancet 2020;395:1569-78.

7 Wölfel R, Corman VM, Guggemos W et al. Virological assessment of hospitalized patients with COVID-2019. Nature 2020;581: 465-69.

8 Beigel JH, Tomashek KM, Dodd LE et al. Remdesivir for the treatment of Covid-19 - preliminary report. N Engl J Med 2020, in press (doi: 10.1056/NEJMoa2007764).

9 Zhou F, Yu T, Du R et al. Clinical course and risk factors for mortality of adult inpatients with COVID-19 in Wuhan, China: a retrospective cohort study. Lancet 2020;395:1054-62.

10 Goldman JD, Lye DCB, Hui DS et al. Remdesivir for 5 or 10 days in patients with severe Covid-19. N Engl J Med 2020, in press (doi: 10.1056/NEJMoa2015301)

11 Olender SA, Perez KK, Go AS et al. Remdesivir for severe COVID-19 versus a cohort receiving standard of care. Clin Infect Dis 2020, in press (doi: 10.1093/cid/ciaa1041).

12 Spinner CD, Gottlieb RL, Criner G] et al. Effect of remdesivir vs standard care on clinical status at 11 days in patients with moderate COVID-19: a randomized clinical trial. JAMA 2020, in press (doi: 10.1001/jama.2020.16349).

13 Department of Health and Social Care. Interim clinical commissioning policy: Remdesivir for patients hospitalised with COVID-19 (adults and children aged 12 years and older). www.england.nhs. uk/coronavirus/wp-content/uploads/sites/52/2020/07/C0654uk-interim-clinical-commissioning-policy-remdesivir-for-patientshospitalised-with-covid-19-adults-and-c.pdf [Accessed 29 ]uly 2020].

14 Day M. Covid-19: Experts criticise claim that remdesivir cuts death rates. BMJ 2020;370:m2839.

15 Horby P, Lim WS, Emberson JR et al. Dexamethasone in hospitalized patients with Covid-19 - preliminary report. N Engl J Med 2020, in press (doi: 10.1056/NEJMoa2021436).

Address for correspondence: Dr Charlotte Richardson, Royal Free London NHS Foundation Trust, Pond Street, London NW3 2QG, UK.

Email: charlotterichardson1@nhs.net 